eine Mondaureole belegt. Das Klein-Stuyvaertsche Schattenphänomen blieb unter diesen Umständen nicht aus. Es wurde ganz in der beschriebenen Weise, bei etwa 30 -facher Teleskopvergrößerung, besonders im zweiten Teil der Mond- finsternis von mir festgestellt und außerdem, unter zeichnerischer Kontrolle, von einer intelligenten, aber in keiner Weise voreingenommenen Hausgenossin.

\title{
Bahnberechnung des Planeten (502) [1903 LC].
}

Aus der letzten Opposition des Planeten (5०2) liegen bisher nur vier Beobachtungen (Rom, A. N. 3985) vor, die den folgenden Normalort ergeben:

$$
\begin{aligned}
& \text { wahre } \alpha \text { 1904.0 wahre } 8 \text { I } 904.0 \\
& \text { I } 904 \text { Juli } 4.5 \quad 8^{\mathrm{h}} \mathrm{r}^{\mathrm{m}} 4^{\mathrm{s}} 45+\mathrm{I}^{\circ} 20^{\prime} 53^{\prime \prime 06} \text {. }
\end{aligned}
$$

Im Jahre 1903 sind sechs Beobachtungen zu Wien (A. N. 3953) gemacht, von denen die ersten vier zu je zwei zu folgenden Orten zusammengezogen sind:

$$
\begin{aligned}
& \alpha 1903.0 \quad \delta 1903.0 \\
& \text { I } 903 \text { Jan. } 31.05^{\mathrm{h}} 3^{8^{\mathrm{m}_{2}} 8^{\mathrm{s}} .05}+3^{\circ} 19^{\prime} 5^{1 .} .8 \\
& \text { Febr. 22.0 } 5412.76 \text { + } 102147.2 \text {. }
\end{aligned}
$$

Diese Koordinaten sind scheinbare zur angegebenen Zeit plus Lichtzeit.

Unter Hinzuziehung der Einzelbeobachtungen 1903 März 5 und 18 (Wien) und mit Berücksichtigung der Jupiter- und Saturnstörungen habe ich auf bekanntem Wege die Verbesserung meiner ersten Bahn (A.N. 3876 ) bestimmt. Mit diesen genaueren Elementen rechnete ich sodann die Störungen neu, und die nochmalige Fehlerausgleichung konnte auf Variation von $\mu$ um +0."०० 28 beschränkt werden, was ohne Einfluß auf die Störungswerte bleibt. In den Bedingungsgleichungen erscheint $\mu$ als unsicherste Unbekannte, und die möglichen Veränderungen der andern Elemente als Funktionen von $A \mu$ sind (logarithmisch, Bezeichnungen nach Bauschinger):

$$
\begin{aligned}
\Delta M_{0} & =1.3087 \mathrm{I} \cdot \Delta \mu \\
\Delta \varphi & =2.12916_{\mathrm{n}} \\
\Delta x & =2.35964 \mathrm{n} \\
\Delta \lambda & =1.62278_{\mathrm{n}} \\
\Delta \nu & =1.68137 \mathrm{n}
\end{aligned}
$$

\begin{tabular}{|c|c|c|c|c|c|}
\hline \multicolumn{3}{|c|}{ Zeit } & $\Delta \alpha \cos \delta$ & $\Delta \delta$ & Anzahl \\
\hline 1903 & Jan. & 31.0 & $+0 "$ I & $+\quad I^{\prime \prime} 2$ & 2 \\
\hline & Febr. & 22.0 & -0.1 & -0.5 & 2 \\
\hline & März & $5 . \cdots$ & +1.6 & 2.0 & $\mathbf{I}$ \\
\hline & $\gg$ & I 8. & -2.9 & +1.3 & I \\
\hline 1904 & Juli & $4 \cdot 5$ & -0.1 & +0.1 & 4 \\
\hline 1903 & Jan. & $21 . \cdots$ & -9.9 & -17.7 & I \\
\hline & $»$ & $27 \cdots$ & -6.7 & +4.1 & 1 \\
\hline
\end{tabular}

Das ermittelte Elementensystem lautet:

$$
\begin{aligned}
& \text { Ep. u. Osc.: } 1903 \text { Febr. } 18.0 \\
& M=33^{\circ} 15^{\prime} 33^{\prime \prime} 6 \mathrm{I} \\
& \omega=17 \text { I } 15.87
\end{aligned}
$$

\begin{tabular}{|c|c|c|c|c|c|}
\hline \multicolumn{2}{|c|}{ 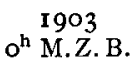 } & $\Delta M$ & $\Delta \omega$ & $\begin{array}{l}\lg (I+\gamma) \\
7 . \text { Dez. }\end{array}$ & 7. $\stackrel{z}{\mathrm{Dez}}$. \\
\hline Jan. & 9 & +0.024 & +0.350 & 3.9 & -4.7 \\
\hline & 19 & +0.011 & +0.196 & 2.4 & -2.8 \\
\hline & 29 & +0.003 & +0.086 & I. 2 & -1.3 \\
\hline Febr. & 8 & 0.000 & +0.021 & 0.3 & -0.4 \\
\hline & I 8 & 0 & 0 & 0 & 0 \\
\hline & 28 & 0.000 & +0.021 & 0.4 & -0.3 \\
\hline März & 10 & -0.002 & +0.083 & I. 6 & -1.4 \\
\hline & 20 & -0.016 & +0.185 & 4.0 & -3.2 \\
\hline & 30 & -0.043 & $+0.3^{2} 3$ & 7.6 & -5 . \\
\hline
\end{tabular}

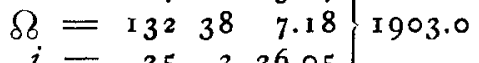

$$
\begin{aligned}
& \varphi=101844.19 \\
& \mu=964.97315 \\
& g=\mathrm{II.2} \quad m_{0}=\text { I } 3.8 \text {. }
\end{aligned}
$$

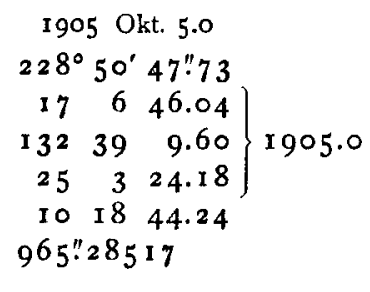

Hiermit werden die genannten Beobachtungen nebst zwei Heidelberger Plattenausmessungen $19{ }^{\circ} 3$ Jan. 2 I und 27 dargestellt $(B-R)$ :

Die photographische Beobachtung 1903 Jan. 21 wird als unsicher bezeichnet.

Bremen, I 905 Januar.
Für eine definitive Rechnung sind in 1903 folgende Störungswerte anzusetzen :

Die Aufsuchungsephemeride für I905 (Opp. Okt. 5) wird dem Königl. Recheninstitut zu Berlin mitgeteilt werden.

\section{Uber einen in BD. nicht vorkommenden Stern $9^{\mathrm{m}} \cdot 0$ nahe BD. $+60^{\circ} \cdot 1358$.}

Als ich am I4. Januar daran ging, den Kometen I904 I zu beobachten, standen in dessen Nähe zwei Sterne $b$ und $c$, von denen $c$, Größe 9.0, der hellste war und darum zum Vergleichstern genommen wurde. Als ich aber nach vollendeter Kometenbeobachtung den Stern $c$ identifizieren wollte, zeigte es sich, daß er in $\mathrm{BD}$. gar nicht vorkommt, während $b$ mit $+60^{\circ} \times 35^{8} 9^{\mathrm{m}} \cdot 5 \mathrm{I}^{\mathrm{h}} 45^{\mathrm{m}} 1^{\mathrm{s}}: 6+60^{\circ} 9^{\prime} 5$ identisch ist. Es war $c-b=-10^{s}+2 \cdot 7$.

Gestern Abend (am I 5. Januar), als es sich eine kleine Weile aufheiterte, sah ich die Gegend wieder nach, und verglich den Stern 6 mit einem Stern $a:$ BD. +60 : ${ }_{3} 6$ I 8.5 I I ${ }^{\mathrm{h}} 46^{\mathrm{m}} 43^{\mathrm{s}} \cdot 3+60^{\circ} 9: 4$. Ich bekam: 